\title{
Effect of Noise on the Average Received SNR of Different Combining Schemes
}

\author{
Shivani Gupta ${ }^{\mathrm{a}^{*}}$, Pragya Awasthi ${ }^{\mathrm{b}}$ \\ ${ }^{a}$ BBDNITM, Lucknow, India \\ ${ }^{b}$ BBDESGI, Lucknow, India
}

\begin{abstract}
In wireless communication system, the multipath fading affects the performance of the system. Diversitycombining scheme is one of the powerful tools to mitigate the effect of fading. The multiple antennas provide an improvement in the capacity and reliability of the system. The performance of four combining schemes Selection Combining (SC), Maximum Ratio Combining (MRC), Equal Gain Combining (EGC), and Generalised Selection combining (GSC)- in the presence of both the perfect and an imperfect channel state information (CSI) at the receiver is studied in this contribution by Monte Carlo simulations in the MATLAB environment, when communicating over Rayleigh fading channels. Our results show that the presence of the complex noise in the channel degrades the performance of the system in all four combining schemes, but the degradation in performance is found to be maximum in MRC and minimum in SC. Performance of EGC lies in between the performance of MRC and SC, whereas the performance of GSC depends upon the number of antennas being selected and it is upper bounded by the MRC and lower bounded by the SC. Also, the results show that as the value of SNR is increased the average received SNR increases in both the channels.
\end{abstract}

Index Terms: Diversity, Rayleigh fading, multipath.

(C) 2014 Published by MECS Publisher. Selection and/or peer review under responsibility of the Research Association of Modern Education and Computer Science

\section{Introduction}

The wireless networks has inherent multipath components leading to multipath fading effects on the communication link`s effective capacity and signal-to-noise-ratio (SNR). But we can exploit multipath scattering by processing multiple signal paths between the transmitter and receiver in order to improve the SNR and channel capacity. This type of signal processing is referred to as Diversity-combining and the wireless channel is termed single-input-multiple-output (SIMO) [1]. The optimum combiner is the MRC whose SNR is the sum of the SNR's of each individual diversity branch. The CSC selects the signal from that diversity branch with the largest instantaneous SNR. The EGC is similar to MRC but it considers the magnitude of all branches unity. The GSC instead of selecting only the branch having the largest instantaneous SNR, as in CSC, it selects 
the $\mathrm{m}$ largest signals from $\mathrm{L}$ total diversity branches and then coherently combines them. After selecting the $\mathrm{m}$ branches we can use either MRC or EGC. Since the schemes require SNR estimation at the receiver, two cases can be explored: perfect channel states information at receiver (CSIR) and imperfect channel states information at receiver. In a separate study [2], we presented the performance of all four combining schemes in the presence of perfect CSIR, and we observed that MRC is the optimal combiner scheme but with added complexity and system cost, while CSC was simpler to implement but provides minimum SNR gain. Also EGC performance is comparable to MRC, while performance of GSC is upper bounded by MRC and lower bounded by CSC.

In this paper for introducing imperfectness in CSI, various level of complex noise is added in complex channel matrix. The performance of all combining schemes deteriorates as the errors were introduced into the estimation of channel coefficients. But the effect is found to be maximum in MRC and minimum in CSC. This is due to the fact that MRC needs perfect estimation of the signal for coherent combination in order to improve link reliability, and for this it needs to track both the phase and amplitude of the signals. Whereas, CSC needs not to track the phase and amplitude since it requires only the measurement of highest SNR. Also, the effect on EGC is comparable to MRC but is less since it tracks only the phase and combines the signals after co-phasing by giving equal weights to all signal paths. The performance of GSC depends upon the selection of number of antennas.

The purpose of this research is to study the feasibility of diversity combining schemes in the wireless communication. Since wireless communication has inherent noise, which cannot be avoided. The results help to determine the selection of combining scheme depending upon the requirement of the application. Various researches have been made in the field of diversity techniques for their practical implementations. The study of FPGA implementation of a supra-optimal receiver diversity combining technique, generalized maximal ratio combining (GMRC) is presented in [1], which can be benefited most by the internet associated wireless peripheral systems. In [3] authors states that the macrodiversity concepts based on MRC have long been applied to the CDMA cellular uplink, mostly in the context of soft handoffs. In [4] authors have proposed a conditional diversity combining scheme, which is called output-threshold maximum ratio combining (OTMRC), which when compared with conventional MRC can significantly reduce the complexity without degrading the required performance. In [5] authors investigate the implementation of antenna diversity in WLAN devices. Though antenna diversity can have various implementations, but these systems are complex and so far have been limited to base station applications. The limitations of antenna selection have been discussed in [6].

\section{System model}

In receiver diversity the multiple copies of the transmitted signals are combined to obtain the resultant signal which is then passed to a standard demodulator. The signals can be combined in different ways. Most combining techniques are linear: the output of the combiner is just a weighted sum of the different fading paths or branches [7]. Fig.1 illustrates the receiver in a diversity combining system.

For combining several branches we need to co-phase them so that they result in the coherent combination of the signals in all the branches. For co-phasing we need to multiply the received signal, $\mathrm{W}_{\mathrm{i}}=\mathrm{a}_{\mathrm{i}} \mathrm{e}^{\mathrm{j} \theta_{\mathrm{i}}}$ with its complex conjugate $W_{i}^{*}=a_{i} e^{-j \theta_{i}}$. At the output of the combiner we get a random SNR $\gamma_{\Sigma}$, whose distribution is a function of the number of diversity paths, the fading distribution on each path and the combining technique.

To study the performance of diversity combining schemes, we have generated the random variables having Rayleigh distribution. The Rayleigh distribution has a probability density function (pdf) given by [8]:

$$
p(r)=\frac{r}{\sigma^{2}} e^{-\frac{r^{2}}{2 \sigma^{2}}} \quad \text { for } r \geq 0
$$




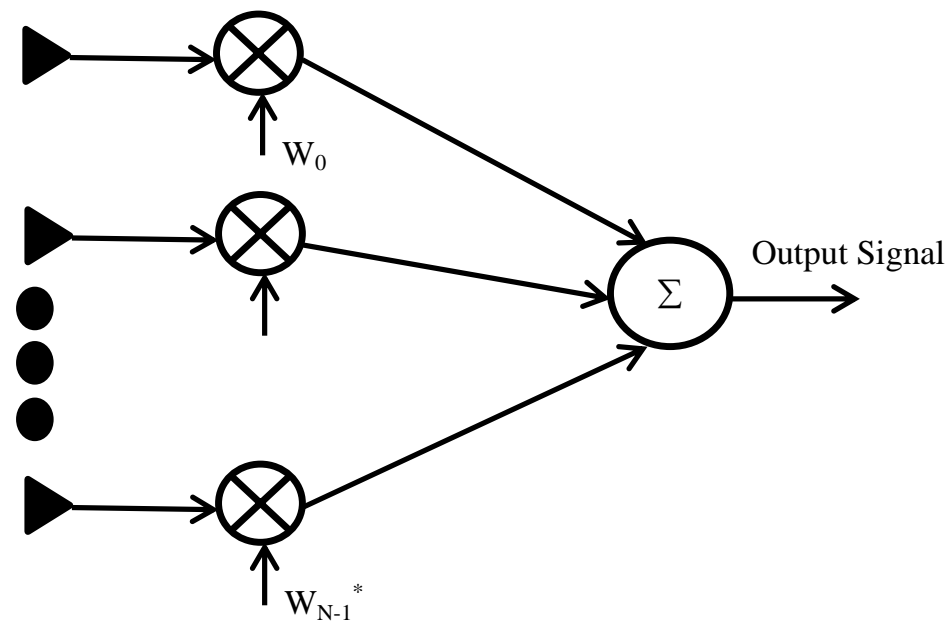

Fig. 1. The receiver in a diversity combining system

Where $\sigma$ is the rms value of the received voltage signal or the standard deviation of the original complex Gaussian signal before envelope detection and $\sigma^{2}$ is the time-average power of the received signal before envelope detrection.

The phase of a Rayleigh variable is uniformly distributed from $[-\pi \pi]$. Thus, pdf of phase is given as:

$$
p(\theta)=\frac{1}{2 \pi} \quad-\pi \leq \theta \leq \pi
$$

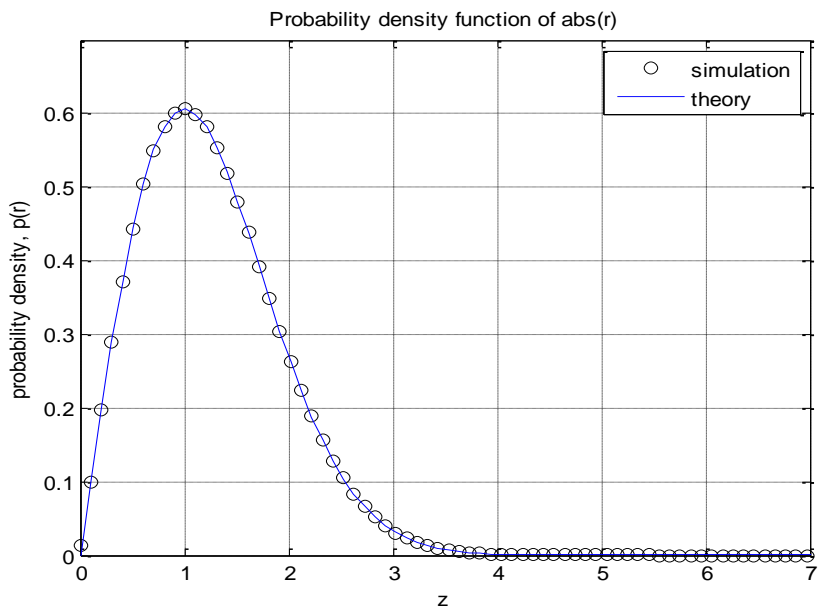

Fig. 2. PDF of amplitude of a Rayleigh variable. 


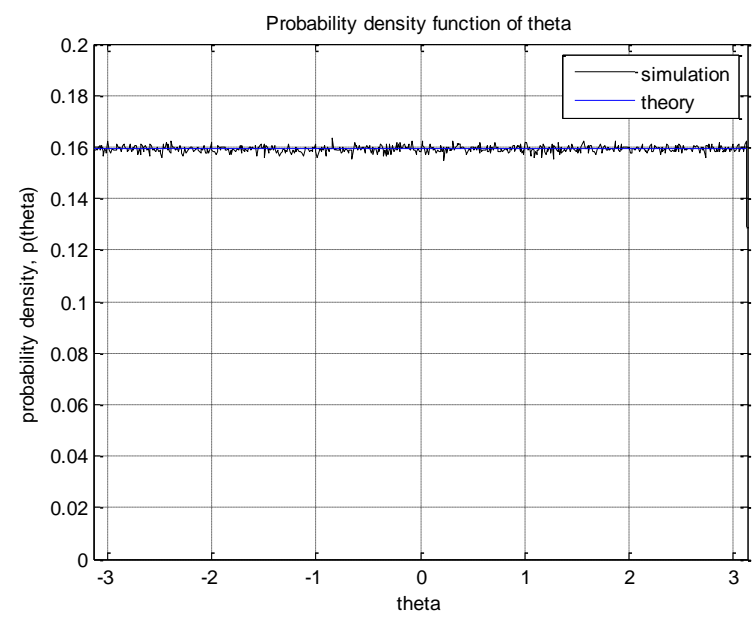

Fig. 3. PDF of phase of a Rayleigh variable.

Fig. 2 and Fig. 3 show the theoretical and simulated pdfs for the amplitude and phase of Rayleigh variable.

In this paper to add imperfectness in the channel various levels of noise are added in the channel matrix $\mathrm{H}$. We can represent the imperfect channel matrix $\mathrm{H}_{\mathrm{N}}$ as:

$$
\mathrm{H}_{\mathrm{N}}=\sqrt{\mathrm{a}} \mathrm{H}+\sqrt{\mathrm{b}} \mathrm{N}
$$

In (2) a and $\mathrm{b}$ denotes the variance of channel matrix and variance of noise matrix respectively. Also, $\mathrm{H}$ and $\mathrm{N}$ are random variables with zero mean and unit variance. The variance of the $\mathrm{H}_{\mathrm{N}}$ should also be unity which implies that $a+b=1$. Thus we can introduce different levels of imperfectness. Referring to Table 1 , the value of $\mathrm{b} / \mathrm{a}$ represents the imperfectness level introduced in the channel.

If $b / a$ is $\infty$, then it indicates that there is no CSI at the receiver, while the value of $b / a=1$ indicates the scenario of perfect estimation.

Table 1. Imperfectness levels

\begin{tabular}{cccc}
\hline S.No. & $\mathbf{a}$ & $\mathbf{b}$ & $\begin{array}{c}\text { Imperfectness } \\
\text { level }(\mathbf{b} / \mathbf{a})\end{array}$ \\
\hline $\mathbf{1}$ & 0 & 1 & $\infty$ \\
$\mathbf{2}$ & 0.25 & 0.75 & 3 \\
$\mathbf{3}$ & 0.50 & 0.50 & 1 \\
$\mathbf{4}$ & 0.75 & 0.25 & 0.33 \\
$\mathbf{5}$ & 1 & 0 & 0 \\
\hline
\end{tabular}

\section{Results}

In our work [2], we have illustrated that as we increase the value of instantaneous SNR, the value of received end-to-end SNR increases in all the combining schemes following the different relationships in the presence of perfect CSIR. Also, we found that the MRC gives the maximum SNR gain and CSC gives the minimum. As we introduce the error in the channel matrix the performance of all the combining schemes starts deteriorating. Fig. 4 shows the condition when we have perfect CSIR. 


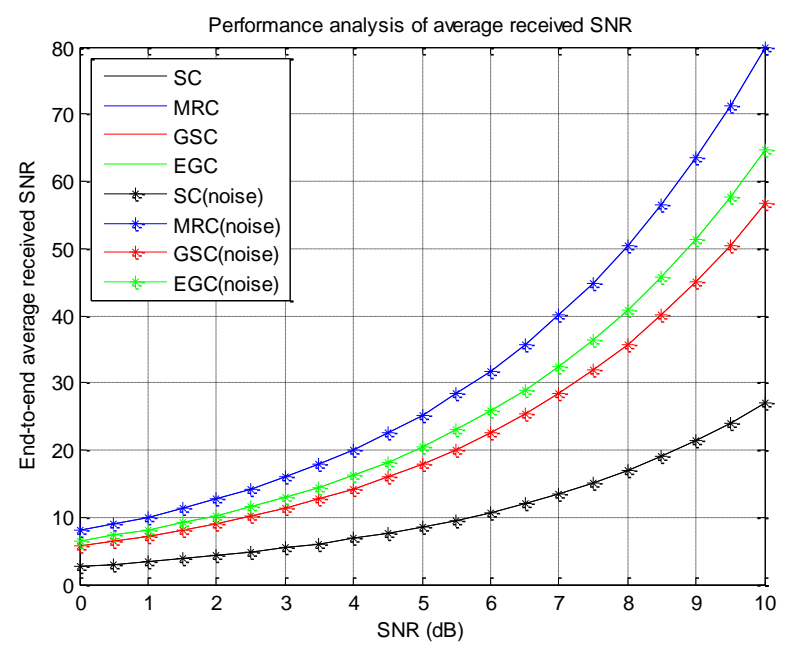

Fig. 4. Average received $\mathrm{SNR}$ for $\mathrm{L}=8, \mathrm{~m}=3$ (for $\mathrm{GSC}$ ), $\mathrm{a}=1$ and $\mathrm{b}=0$.

We added some error in the channel matrix by making $b=0.25$ and $a=0.75$. We find that with the addition of error the performance of combining schemes deteriorates. Fig.5 shows the plots with the added noise. The effect is found to be in the following order:

$$
\mathrm{MRC}>\mathrm{EGC}>\mathrm{GSC}>\mathrm{CSC}
$$

Table 2 shows the values at $\mathrm{SNR}=5 \mathrm{~dB}$. It is evident from the values that the maximum difference in the value of SNR is found to be in MRC.

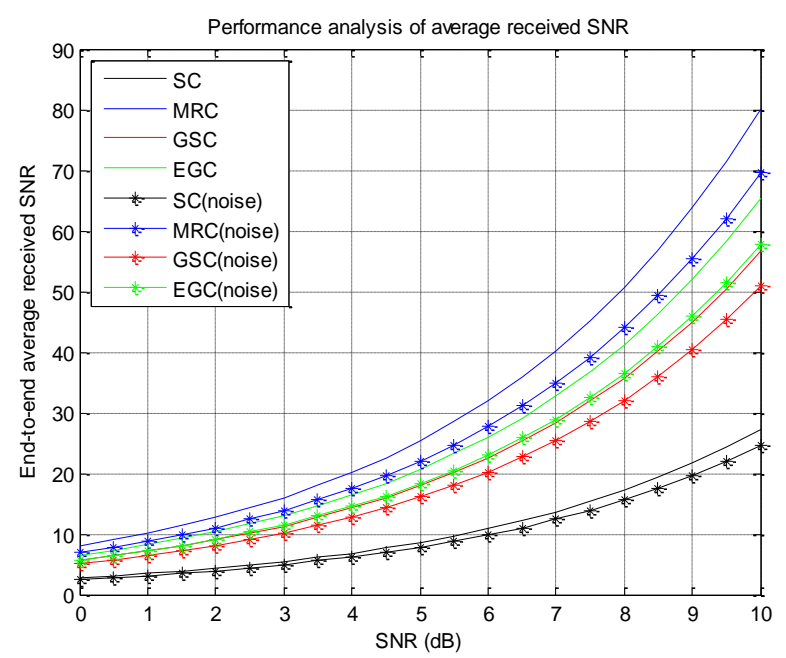

Fig. 5. Average received $\mathrm{SNR}$ for $\mathrm{L}=8, \mathrm{~m}=3$ (for $\mathrm{GSC}$ ), $\mathrm{a}=0.75$ and $\mathrm{b}=0.25$. 
Table 2. $a=0.75$ and $b=0.25$

\begin{tabular}{ccc}
\hline Technique used & At SNR=5dB & Difference \\
\hline SC & 8.601 & 0.795 \\
SC(N) & 7.806 & \\
MRC & 25.37 & 3.33 \\
MRC(N) & 22.04 & \\
GSC & 18.26 & 2.2 \\
GSC(N) & 16.06 & \\
EGC & 20.64 & 2.38 \\
EGC(N) & 18.26 & \\
\hline
\end{tabular}

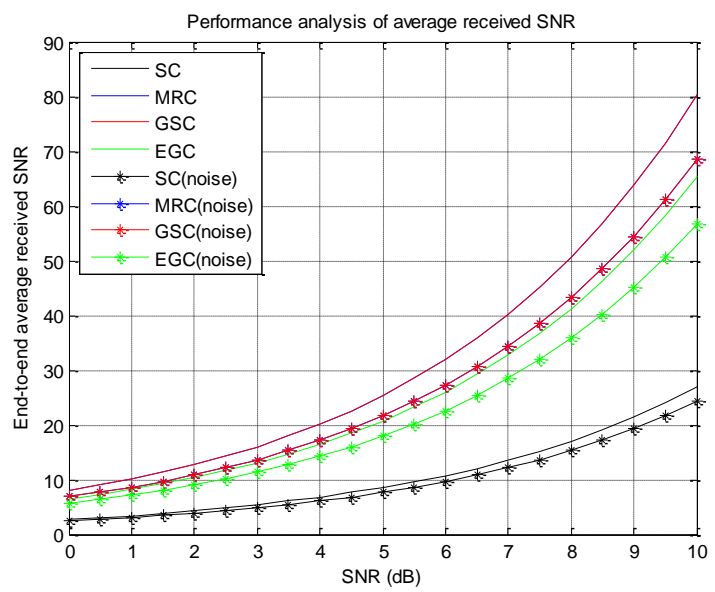

Fig. 6. Average received SNR for $\mathrm{L}=8, \mathrm{~m}=8$ (for GSC), $\mathrm{a}=0.75$ and $\mathrm{b}=0.25$.

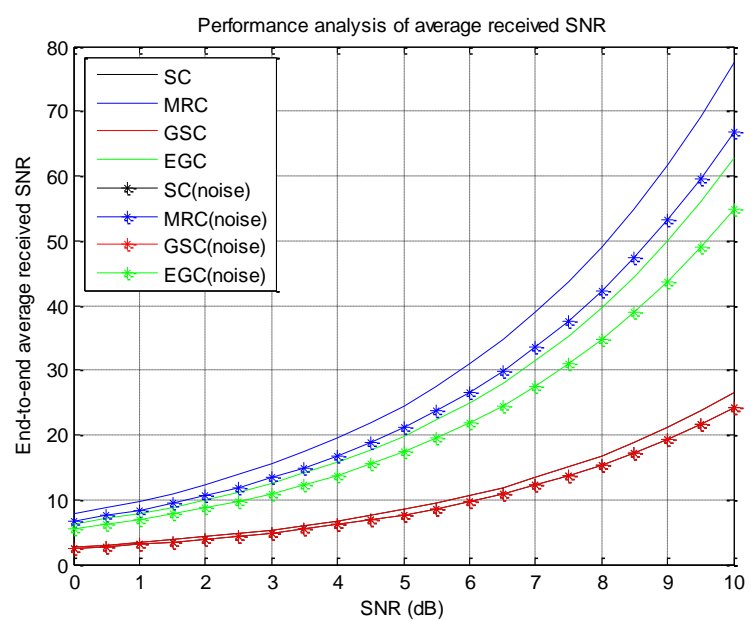

Fig. 7. Average received SNR for $\mathrm{L}=8, \mathrm{~m}=1$ (for $\mathrm{GSC}$ ), $\mathrm{a}=0.75$ and $\mathrm{b}=0.25$. 
Fig. 6 and Fig.7 indicates that the performance of GSC lies in the fact that how many number of antennas are being selected. It is indicative from the plots that GSC is upper bounded by MRC and lower bounded by CSC. In Fig. 8 we have taken both $\mathrm{a}$ and $\mathrm{b}$ as 0.5 , i.e. we have $50 \%$ noise and $50 \%$ signal. The performance is being further deteriorated following the order:

$$
\mathrm{MRC}>\mathrm{EGC}>\mathrm{GSC}>\mathrm{CSC}
$$

Table 3 gives the value of $S N R$ at $5 \mathrm{~dB}$ for $\mathrm{a}=0.5$ and $\mathrm{b}=0.5$.

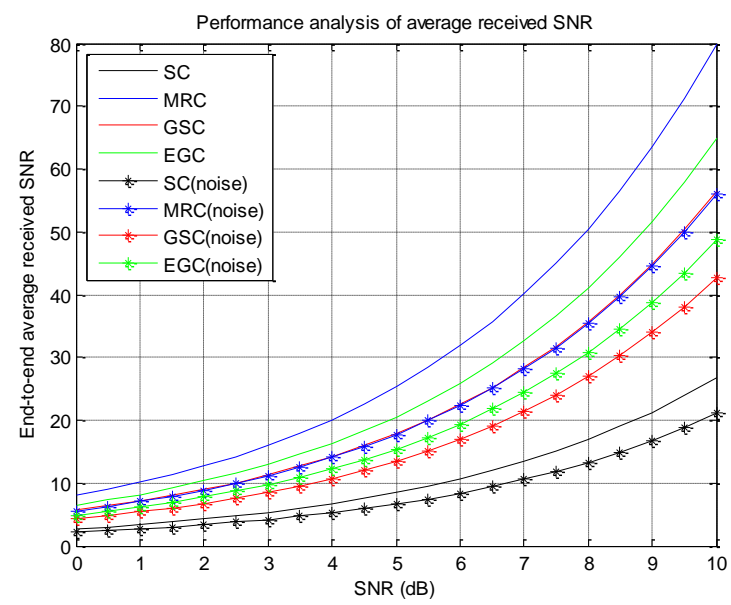

Fig. 8. Average received SNR for $\mathrm{L}=8, \mathrm{~m}=3$ (for GSC), $\mathrm{a}=0.5$ and $\mathrm{b}=0.5$.

Next we have further increased the level of noise by making $b=0.75$ and $a=0.25$. Now, the level of noise is more than the signal. It further deteriorates the performance. Fig. 9 and table 4 gives the results for the same. The order of deterioration in the performance is:

$$
\mathrm{MRC}>\mathrm{EGC}>\mathrm{GSC}>\mathrm{CSC}
$$

Table 3. $a=0.5$ and $b=0.5$

\begin{tabular}{ccc}
\hline Technique used & At SNR=5dB & Difference \\
\hline SC & 8.47 & 1.809 \\
SC(N) & 6.661 & \\
MRC & 25.28 & 7.55 \\
MRC(N) & 17.73 & \\
GSC & 17.73 & 4.2 \\
GSC(N) & 13.53 & \\
EGC & 20.55 & 5.14 \\
EGC(N) & 15.41 & \\
\hline
\end{tabular}




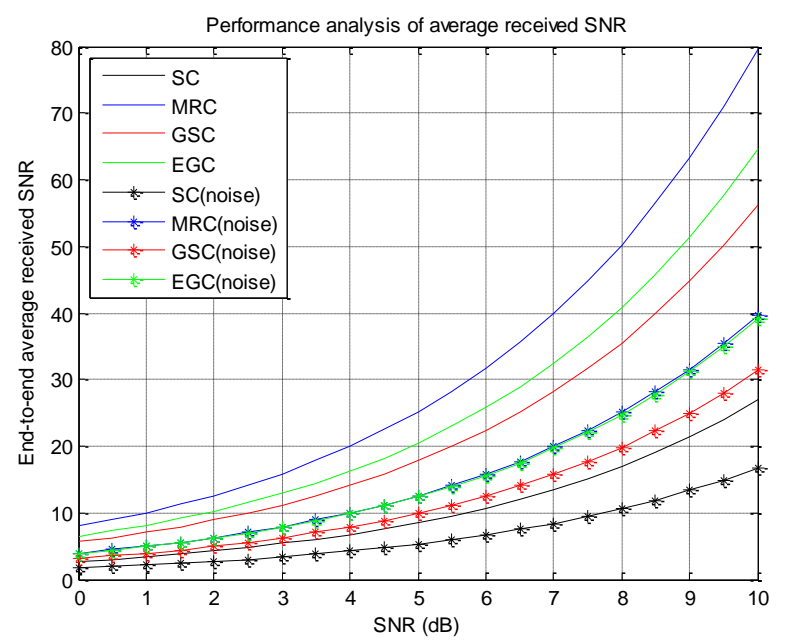

Fig. 9. Average received SNR for $\mathrm{L}=8, \mathrm{~m}=3$ (for GSC), $\mathrm{a}=0.25$ and $\mathrm{b}=0.75$.

Table 4. $a=0.25$ and $b=0.75$

\begin{tabular}{ccc}
\hline Technique used & At SNR=5dB & Difference \\
\hline SC & 8.534 & 3.223 \\
SC(N) & 5.311 & \\
MRC & 25.2 & 12.78 \\
MRC(N) & 12.42 & \\
GSC & 17.79 & 7.848 \\
GSC(N) & 9.942 & \\
EGC & 20.47 & 8.05 \\
EGC(N) & 12.42 & \\
\hline
\end{tabular}

Next we consider that we have no CSI at the receiver. Thus, we have the maximum error in the received signal. This condition is achieved by making $a=0$ and $b=1$. The performance deteriorates appreciably in all the techniques. Fig. 10 and table 5 shows the results for the same. The order in which performance deteriorates is:

$$
\mathrm{MRC}>\mathrm{EGC}>\mathrm{GSC}>\mathrm{CSC}
$$

\section{Conclusions}

In this paper we have generated random variables having Rayleigh distribution and then implemented the different combining schemes. Then we studied the effect of addition of Gaussian noise to the channel matrix. To introduce imperfectness in the channel we have introduced different levels of noise to the channel matrix. Results for different values of $\mathrm{a}$ and $\mathrm{b}$ are presented. Both the condition of perfect CSI and imperfect CSI are studied. We found that though MRC gives the highest SNR gain, the effect of noise is maximum in MRC. While CSC gives the least SNR gain but the effect of noise is found to be minimum. EGC performance is comparable to MRC. Performance of GSC varies between MRC and CSC, depending upon the number of antennas selected. In the case of no CSI the result is found to be worse. Thus to have the improvement in the 
link reliability we need to have perfect estimation of channel state. If we have perfect CSIR then MRC will be the optimal combiner scheme, but if we have lower value of CSIR or no CSIR, we must prefer to implement CSC. Also if we want SNR gain as well as less effect of noise then we must go for the implementation of either EGC or GSC, performance of GSC being subjected to the number of antennas being selected. All the results are evaluated with the help of Monte Carlo simulations in the MATLAB environment. It is expected that work presented in this paper will be helpful for incorporating diversity schemes in communication system.

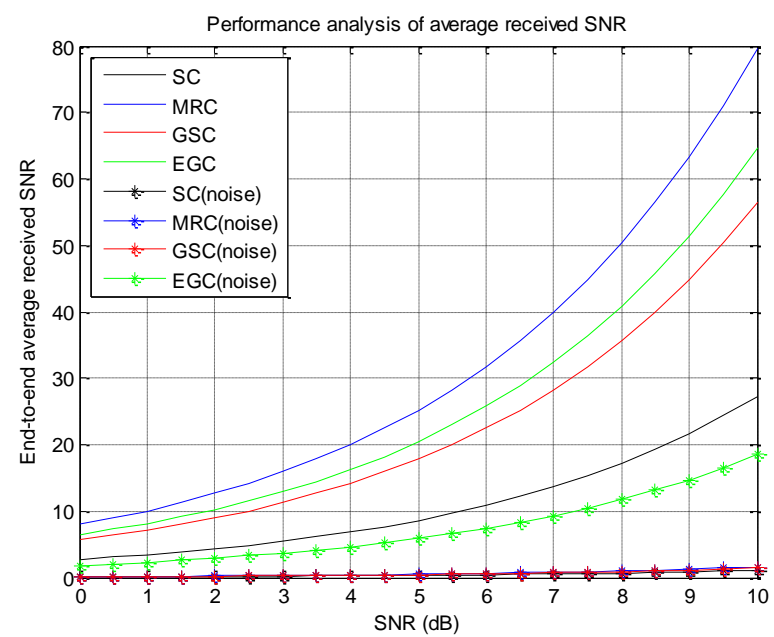

Fig. 10. Average received SNR for $\mathrm{L}=8, \mathrm{~m}=3$ (for GSC), $\mathrm{a}=0$ and $\mathrm{b}=1$

Table 5 . $a=0$ and $b=1$

\begin{tabular}{lll}
\hline Technique used & At SNR=5dB & Difference \\
\hline SC & 8.642 & 8.29 \\
SC(N) & 0.3489 & \\
MRC & 25.21 & 24.71 \\
MRC(N) & 0.4983 & \\
GSC & 17.85 & 17.38 \\
GSC $(N)$ & 0.4642 & \\
EGC & 20.46 & 14.6 \\
EGC(N) & 5.863 & \\
\hline
\end{tabular}

\section{References}

[1] Rafic Ayoubi, Jean-Pierre Dubois, Rania Minkara, "FPGA Implementation of Generalized Maximal Ratio Combining Receiver Diversity", World Academy od Science, Engineering and Technology, 44, 2010.

[2] Shivani Gupta, Dr. Himanshu Katiyar, "Comparative Study of Average Received SNR of Different Combining Schemes", ISRTEC-2012, KNIT, Sultanpur.

[3] A. Viterbi, A. Viterbi, K. Gilhousen, and E. Zehavi, "Soft handoff extends CDMA cell coverage and increases reverse link capacity,” IEEE J. Select. Areas Commun., vol. 12, pp. 1281-1288, Oct. 1994. 
[4] Haewoon Nam; Young-Chai Ko; Womack, B.F., "Performance Analysis of OT-MRC Over I.I.D. Nakagami and Non-I.I.D. Rayleigh Fading Channels", IEEE transaction, vol. 45, issue 6, pp. 1941-1946, 2006.

[5] Frank M. Caimi, Kerry L. Greer, Jason M. Hendler, “Antenna Diversity in Wireless Local Area Network Devices", Jan 2002.

[6] Shahab Sanayei, Aria Nosratinia, "Antenna Selection in MIMO Systems", IEEE commun. Magazine, Oct. 2004.

[7] Andrea Goldsmith, "Wireless Communications", Stanford University, Cambridge University Press, pp. 191-192.

[8] Theodore S. Rappaport, "Wireless Communications", second edition, pp. 210.

\section{Author(s) Profile}

Shivani Gupta has obtained her B.Tech degree in 2009 and is presently working as a lecturer in BBDNITM, Lucknow. She is presently pursuing her M.Tech (Part - time) from BBDU, Lucknow. Her area of interest is in wireless communications and channel modeling.

Pragya Awasthi has obtained her B.Tech degree in 2009 and is presently working as a lecturer in BBDESGI, Lucknow. She is presently pursuing her M.Tech (Part - time) from BBDU, Lucknow. Her area of interest is in wireless communications.

How to cite this paper: Shivani Gupta, Pragya Awasthi,"Effect of Noise on the Average Received SNR of Different Combining Schemes ", IJWMT, vol.4, no.1, pp.50-59, 2014.DOI: 10.5815/ijwmt.2014.01.04 